\title{
PROGRAMAS DE EXERCÍCIO FÍSICO COM JOGOS PRÉ- DESPORTIVOS PARA CRIANÇAS COM SOBREPESO E OBESIDADE
}

\author{
Bruno de Souza Vespasiano \\ Universidade Metodista de Piracicaba, Piracicaba, São Paulo, Brasil. \\ Joelma Lirane Pontes da Mota \\ Faculdade de Ciências Sociais e Agrárias de Itapeva, Itapeva, São Paulo, Brasil. \\ Marcelo de Castro Cesar \\ Universidade Metodista de Piracicaba, Piracicaba, São Paulo, Brasil.
}

\begin{abstract}
Resumo
Objetivou-se verificar os efeitos de um programa de exercícios (PEF) com jogos préesportivos atletismo, voleibol e basquetebol em crianças com sobrepeso e obesidade. Participaram 74 crianças, agrupadas em GC $(n=36)$ e GI $(n=38)$. Os voluntários foram submetidos a avaliações antropométricas e avaliações das capacidades físicas $(\mathrm{CF})$, pré e pós 52 semanas. Todos frequentavam aulas de Educação Física escolar, e o GI participou do PEF. No GC, não ocorreram diferenças significativas nas variáveis indicadoras da adiposidade (VIA), houve piora na agilidade e na velocidade, sem alterações significativas em outras CF. No GI, houve redução de VIA e melhora das $\mathrm{CF}$, exceto resistência abdominal. Conclui-se que o PEF proporcionou benefícios aos voluntários do GI.
\end{abstract}

Palavras-chave: Crianças. Aptidão física. Exercício. Obesidade.

\section{EXERCISE PROGRAMS WITH PRE-SPORTS GAMES FOR OVERWEIGHT AND OBESE CHILDREN}

\begin{abstract}
The objective of this study was to verify the effects of an exercise program (PEF) with presports athletics, volleyball and basketball in overweight and obese children. Participants were 74 children, grouped in CG $(n=36)$, and GI $(n=38)$. The volunteers were submitted to anthropometric assessments and physical abilities (CF) assessments, pre and post 52 weeks. All attended school Physical Education classes, and the GI participated in the PEF. In the CG, there were no significant differences in the adiposity indicators (VIA), agility and speed worsening, without significant changes in other CF. In the GI, there was reduction of VIA and improvement of the $\mathrm{CF}$, except abdominal resistance. It was concluded that the PEF provided benefits to the volunteers of the GI.
\end{abstract}

Keywords: School. Physical fitness. Overweight. Obesity.

PROGRAMAS DE EJERCICIO FÍSICO CON JUEGOS PRE-DEPORTIVOS PARA NIÑOS CON SOBREPESO Y OBESIDAD

\section{Resumen}

Se objetivó verificar los efectos de un programa de ejercicios (PEF) con juegos pre-deportivos atletismo, voleibol y baloncesto en niños con sobrepeso y obesidad. Participaron 74 niños, 
agrupados en GC $(n=36)$, y GI $(n=38)$. Los voluntarios fueron sometidos a evaluaciones antropométricas y evaluaciones de las capacidades físicas (CF), pre y post 52 semanas. Todos frecuentaban clases de Educación Física escolar, y el GI participó en el PEF. En el GC no hubo diferencias significativas en las variables indicadoras de la adiposidad (VIA), hubo empeoramiento en la agilidad y la velocidad, sin cambios significativos en otras CF. En el GI hubo reducción de VIA y mejora de las CF, excepto resistencia abdominal. Se concluye que el PEF proporcionó beneficios a los voluntarios del GI.

Palabras clave: Escolares. Aptitud física. Sobrepeso. Obesidad.

\section{Introdução}

Em países desenvolvidos, assim como aqueles que estão em desenvolvimento, a obesidade é considerada grave problema de saúde pública. Nos últimos 30 anos, devido à maior oferta de alimentos, dentre eles os industrializados, e a constante diminuição da prática de exercícios físicos, têm-se observado aumentos gradativos de obesidade que geram impactos negativos na saúde e diminui a qualidade de vida dos indivíduos (BRASIL, 2008). Devido à mudança de hábitos de vida, atualmente, o Brasil encontra-se na transição da desnutrição para a obesidade, uma vez que a prevalência de excesso de peso corporal (sobrepeso e obesidade) atingiu 53,8\% da população em 2016 (ABESO, 2009; BRASIL, 2016).

Sobre isso, a Sociedade Brasileira de Pediatria (2015) aponta que a obesidade é considerada epidêmica e tem origem multifatorial além de estar relacionada a fatores comportamentais, genéticos e ambientais que podem desencadear diversas patologias comprometendo à saúde. Quando observado em crianças e adolescentes, os índices de obesidade e problemas decorrentes são próximos aos que ocorrem em adultos como, por exemplo, o diabetes tipo II, hipertensão arterial, doenças cardiovasculares, além da queda do rendimento escolar e dificuldades de socialização (OLIVEIRA; FISBERG, 2003; OLIVEIRA et al., 2003; LIMA et al., 2004; OLIVEIRA et al., 2004; ALVES, 2008; SOCIEDADE BRASILEIRA DE PEDIATRIA; 2015).

Crianças e adolescentes passam mais tempo em frente à televisão e utilizam com maior frequência os recursos tecnológicos o que culmina em diminuição da prática de exercício físico e gasto calórico resultando em maior acúmulo de gordura corporal (AMARAL; PALMA, 2001; MOTA et al., 2015). Nesse sentido, a Educação Física escolar tem um papel importante na educação e consequente formação dos alunos. Atualmente, a Educação Física, dentre suas propostas, tem condições de desenvolver trabalhos voltados à saúde, qualidade de vida e promoção da saúde (BRASIL, 2012).

Assim, a Educação Física, em consonância com as suas finalidades, deve propor práticas corporais como esportes, atividades rítmicas, jogos, lutas e ginástica além de oferecer amplo desenvolvimento nos aspectos motores, cognitivos e sociais - afetivos (PROPOSTA CURRICULAR DO ESTADO DE SÃO PAULO, 2008; SILVA et al., 2011).

O exercício físico deve compor as aulas de educação física atendendo às necessidades e aos respectivos planejamentos escolares além de oferecer benefícios aos seus praticantes (ACSM, 1997; DARIDO, 2003; PROPOSTA CURRICULAR DO ESTADO DE SÃO PAULO, 2008; SILVA et al., 2011; ACSM, 2014; SOCIEDADE BRASILEIRA DE PEDIATRIA, 2015).

O professor de educação física que atua também na saúde deve incentivar e proporcionar programas de exercícios físicos que visem à criação de hábitos saudáveis e diminuição da obesidade em crianças e adolescentes (BRASIL, 2008; BRASIL, 2009; BRASIL, 2012). Dado o contexto, este estudo tem como objetivo analisar os efeitos de um programa de exercí- 
cios com jogos pré-desportivos na antropometria e nas capacidades físicas de crianças com sobrepeso e obesidade.

\section{Materiais e Métodos}

Trata-se de uma pesquisa experimental com duração de 52 semanas, realizada no município de Nova Campina. Esse município é localizado no interior do Estado de São Paulo, possui 8.515 habitantes, desse total, 1711 são alunos matriculados no ensino fundamental e distribuídos em sete unidades escolares municipais, em que quatro unidades estão localizadas na zona urbana e outras três na zona rural do município. Na cidade, não há rede particular de ensino (IBGE, 2010).

Foram selecionadas crianças do sexo feminino e masculino de acordo com os seguintes critérios de inclusão: idade entre 6 e 8 anos, índice de massa corporal (IMC) maior que percentil 85 de acordo com a WHO (2007) e participantes das duas aulas semanais de educação física escolar.

Os critérios de exclusão para os dois grupos foram: crianças com queixas ou doenças que contraindicassem a prática de exercícios físicos e participantes de outros projetos com prática regular de exercícios. Para o grupo intervenção (GI), os voluntários que não tivessem frequência superior a $75 \%$ das aulas do projeto seriam excluídos.

Após a triagem de 540 alunos do primeiro ciclo do ensino fundamental, 177 alunos estavam com idade entre 6 e 8 anos e 11 meses, em que 74 deles apresentavam sobrepeso ou obesidade, que foram incluídos na amostra da pesquisa.

Os 74 voluntários foram agrupados em: Grupo Controle (GC) com 36 voluntários, idade de 7,31 \pm 0,67 anos, sendo 19 meninas e 17 meninos, 10 com obesidade e 26 com sobrepeso, que realizaram apenas duas aulas de Educação Física escolar, com duração de 50 minutos, e não participaram de outros programas de exercícios físicos; Grupo Intervenção (GI) com 38 voluntários, idade de 7,13 \pm 0,63 anos, 23 meninas e 15 meninos, sendo 16 com obesidade e 22 com sobrepeso, que participaram do programa de exercícios físicos, além das duas aulas semanais de Educação Física escolar com duração de 50 minutos.

Os responsáveis responderam a um questionário do estado de saúde das crianças, para descartar contraindicações à prática de exercícios físicos, e assinaram Termo de Consentimento Livre e Esclarecido, autorizando participação no projeto. O presente estudo foi aprovado pelo Comitê de Ética de Pesquisa da Universidade Metodista de Piracicaba com protocolo $\mathrm{n}^{\mathrm{o}}$ 49/13.

O protocolo experimental iniciou-se em agosto de 2014 e encerrou-se em setembro de 2015. As etapas do programa de exercícios foram estabelecidas da seguinte forma: triagem prévia dos alunos; seleção dos voluntários; reuniões com a diretora do departamento de educação do município; reuniões com os responsáveis pelos alunos; avaliações e programa de exercícios físicos.

\section{Avaliação antropométrica e das capacidades físicas}

Foram realizadas avaliações pré e pós 52 semanas. Os voluntários foram orientados a não realizar qualquer tipo de exercício físico nos dias das avaliações. Além disso, eles receberam informações sobre a utilização de roupas adequadas e foi pedido a eles que evitassem hábitos que pudessem interferir no desempenho de cada um deles. Por fim, as avaliações foram realizadas no período da manhã.

As medidas antropométricas foram peso corporal, estatura, envergadura, circunferências da cintura, circunferência do braço direito e dobra cutânea tricipital direita. Para a aferição do peso corporal e da estatura, foi utilizada uma balança Filizola® com estadiômetro aco- 
plado a ela. O IMC foi calculado pelo peso corporal em quilogramas dividido pela estatura em metros ao quadrado, e os alunos foram classificados a partir das recomendações da WHO (2007), sendo sobrepeso os percentis acima de 85 até 97 e obesidade acima de 97 . Na envergadura, uma fita métrica foi anexada a uma parede plana de forma paralela ao solo (PROESPBR, 2012). Para aferição na circunferência da cintura, uma fita métrica foi colocada no ponto médio entre a última costela e a crista ilíaca (ABESO, 2009) e na circunferência do braço, a fita métrica foi colocada na região mesoumeral do braço direito (FRISANCHO, 1990). A dobra cutânea tricipital do braço direito foi aferida com um compasso da marca Cescorf®, na distância média entre a borda lateral do acrômio e o olecrano (FRISANCHO, 1990; COSTA, 2001).

Para a realização dos testes das capacidades físicas, foram seguidas as recomendações do PROESP-BR (2012): sentar e alcançar (para avaliação da flexibilidade), resistência abdominal (resistência muscular localizada abdominal), salto horizontal (força explosiva de membros inferiores), arremesso de medicineball (força explosiva de membros superiores), quadrado (agilidade), corrida de 20 metros (velocidade) e teste de corrida/caminhada de seis minutos (aptidão cardiorrespiratória), além das tabelas (PROESP-BR) de referência para posteriores comparações.

\section{Programa de exercícios físicos}

As aulas ocorreram três vezes por semana (segundas, quartas e sextas-feiras) com duração de 60 minutos cada uma delas. Nas duas primeiras semanas de agosto de 2014, foram realizadas as avaliações dos voluntários (pré-programa). A partir da terceira semana do mesmo mês, os alunos foram submetidos a 15 semanas de práticas corporais, utilizando o atletismo como jogo pré-desportivo até o mês de novembro de 2014 (GONZÁLES, DARIDO, OLIVEIRA, 2014). Após o período de férias de quatro semanas, os voluntários retornaram ao projeto na última semana de janeiro de 2015, sendo proporcionadas 15 semanas do jogo prédesportivo voleibol, até a primeira semana de maio de 2015. Logo a seguir, 15 semanas do jogo pré-desportivo basquetebol até a última semana de agosto de 2015. Na semana seguinte, foram realizadas as avaliações antropométricas e das capacidades físicas (pós-programa).

As sessões do programa foram divididas em 15 semanas de atletismo que culminaram em 45 sessões de exercícios, sendo 15 delas destinadas a exercícios que trabalhavam a corrida especificamente considerada uma habilidade básica com foco na melhora da resistência. Em outras 15 sessões, além da corrida, foi desenvolvida força de membros inferiores com exercícios que envolviam saltos, agachamentos e saltos unilaterais de forma lúdica ao ponto de desenvolverem força de membro inferior. Nas últimas 15 sessões do atletismo, o foco foi em exercícios de potência anaeróbia com aulas que estimulavam os alunos a correrem com maior velocidade em distancias de 20 metros para melhorarem a capacidade de força rápida.

As 45 sessões de voleibol foram divididas de acordo com as recomendações de aulas propostas por Gonzáles, Darido, Oliveira (2014). Destaca-se que as 15 sessões foram destinadas aos jogos reduzidos. Outras 15 sessões foram destinadas a atividades destinadas aos jogos adaptados ao ponto que os voluntários também desenvolviam força de membro superior devido às características da modalidade. As últimas 15 sessões foram voltadas ao trabalho de agilidade e potência de membros inferiores em que o foco foi desenvolver melhoras nas capacidades já trabalhadas.

Nas últimas 45 sessões do projeto, desenvolveu-se a modalidade de basquete, uma vez que esse esporte propicia a coordenação motora, lateralidade, agilidade, além de melhorar a força. As 15 primeiras sessões, os alunos eram incentivados a conhecer a modalidade fazendo os jogos reduzidos a fim de trabalhar capacidades como velocidade, agilidade, entre outras. Nas 15 sessões subsequentes, os voluntários praticaram exercícios com bola de modo a me- 
lhorar a coordenação motora e, nas 15 sessões finais, eles faziam exercícios que incentivavam trabalho de força de membros inferiores e superiores.

Durante todo o programa de exercícios, sessões de alongamento também eram propostas aos voluntários com intuito de melhorar a flexibilidade (SOCIEDADE BRASILEIRA DE MEDICINA DO ESPORTE, 1998).

\section{Análise Estatística}

Os dados estão apresentados em média e desvio padrão. Para verificar os pressupostos de normalidade, foi realizado o teste de Shapiro-Wilk. Para comparar os momentos pré e pós período experimental dos GC e GI, foi realizada a Anova Two Way.

O nível de significância adotado foi 5\% (p < 0,05). O software Graph Pad Prism versão 6.0 foi utilizado para realização dos cálculos.

\section{Resultados}

Os 74 voluntários completaram todas as atividades planejadas. No grupo intervenção, a frequência foi superior a $75 \%$ em todos os voluntários e não ocorreram intercorrências durante os testes e o programa de exercícios físicos. Destaca-se que os pais e os alunos se mostraram muito interessados em que os alunos pudessem participar do programa de exercícios físicos.

Quando analisados GC e GI nos testes iniciais, antes das 52 semanas da intervenção, não foram encontradas diferenças significativas.

Na comparação das variáveis antes e após as 52 semanas, no GC, não houve diferenças significativas na estatura (pré 1,28 $\pm 0,09 \mathrm{~m}$, pós $1,29 \pm 0,08 \mathrm{~m}$ ), na envergadura (pré $147,33 \pm 6,00 \mathrm{~cm}$, pós $148,92 \pm 5,36 \mathrm{~cm}$ ) e na circunferência do braço direito (pré 21,53 \pm $1,80 \mathrm{~cm}$, pós $21,58 \pm 1,46 \mathrm{~cm})$.

No GI, não houve diferenças significativas na estatura (pré 1,25 $\pm 0,07 \mathrm{~m}$, pós 1,26 \pm $0,07 \mathrm{~m}$ ), ocorreu aumento ( $\mathrm{p}<0,05$ ) na envergadura (pré 146,53 $\pm 3,10 \mathrm{~cm}$, pós $148,29 \pm 2,91$ $\mathrm{cm}$ ) e diminuição $(\mathrm{p}<0,05)$ na circunferência do braço direito (pré 20,92 $\pm 1,58 \mathrm{~cm}$, pós $19,79 \pm 1,72 \mathrm{~cm})$.

Na Tabela 1 encontram-se os resultados das variáveis antropométricas indicadoras da adiposidade corporal do GC e GI pré e pós 52 semanas. Para o GC, os resultados não indicaram diferenças significativas nas seguintes variáveis: massa corporal, índice de massa corporal, circunferências da cintura e dobra cutânea tricipital. No GI, os resultados indicaram diminuição nas variáveis: massa corporal, índice de massa corporal, circunferências da cintura e dobra cutânea tricipital. 
Tabela 1. Média, desvio padrão e resultados da análise estatística da antropometria dos voluntários do GC e GI pré e pós período experimental.

\begin{tabular}{lcccc}
\hline Variáveis & Pré & Pós & Pré & Pós \\
& & & & \\
Massa Corporal (Kg) & $32,98 \pm 7,55$ & $33,06 \pm 7,33$ & $33,58 \pm 8,34$ & $32,05 \pm 8,18^{*}$ \\
IMC $\left(\mathrm{Kg} / \mathrm{m}^{2}\right)$ & $20,15 \pm 2,40$ & $20,18 \pm 2,35$ & $21,37 \pm 3,78$ & $19,91 \pm 3,70^{*}$ \\
& & & & \\
Circ. Cintura (cm) & $63,89 \pm 2,48$ & $64,67 \pm 2,49$ & $63,39 \pm 2,02$ & $61,08 \pm 1,77^{*}$ \\
& & & & \\
DC Tricipital (D) & $15,03 \pm 1,77$ & $16,47 \pm 1,66$ & $14,63 \pm 1,60$ & $12,34 \pm 1,72^{*}$
\end{tabular}

MC- Massa Corporal; IMC - índice de massa corporal; Circ. Cintura - circunferência da cintura; DC tricipital D - Dobra Cutânea Tricipital Direita. *p $<0,05$.

$\mathrm{Na}$ Tabela 2 encontram-se os resultados dos testes das capacidades físicas do GC e GI pré e pós 52 semanas. No GC, não houve diferenças significativas nos testes sentar e alcançar, resistência abdominal, salto horizontal, arremesso de medicineball e corrida/caminhada dos seis minutos, houve aumento no tempo dos testes quadrado e corrida de 20 metros. No GI, por sua vez, houve aumento na distância atingida no teste de sentar e alcançar, salto horizontal, arremesso de medicineball e corrida/caminhada de seis minutos e ocorreu redução no tempo dos testes quadrado e corrida de 20 metros, não houve diferença significativa no teste de resistência abdominal. 
Tabela 2. Média, desvio padrão e resultados da análise estatística das capacidades físicas dos voluntários do GC e GI pré e pós período experimental.

\begin{tabular}{|c|c|c|c|c|}
\hline \multicolumn{3}{|c|}{ Grupo Controle } & \multicolumn{2}{|c|}{ Grupo Intervenção } \\
\hline Variáveis & Pré & Pós & Pré & Pós \\
\hline Sentar e alcançar $(\mathrm{cm})$ & $30,74 \pm 4,15$ & $30,88 \pm 3,40$ & $28,59 \pm 4,96$ & $29,78 \pm 5,03^{*}$ \\
\hline $\begin{array}{l}\text { Resistência abdominal } \\
\text { (repetições) }\end{array}$ & $18,33 \pm 6,96$ & $17,67 \pm 9,97$ & $17,84 \pm 6,13$ & $18,66 \pm 5,23$ \\
\hline Salto Horizontal (cm) & $95,67 \pm 22,55$ & $94,97 \pm 21,78$ & $92,87 \pm 16,47$ & $98,74 \pm 17,80^{*}$ \\
\hline $\begin{array}{l}\text { Arremesso Medicineball } \\
(\mathrm{cm})\end{array}$ & $140,47 \pm 23,06$ & $140,58 \pm 21,78$ & $129,55 \pm 29,91$ & $138,18 \pm 21,29 *$ \\
\hline Quadrado (segundos) & $8,47 \pm 0,70$ & $9,32 \pm 1,14^{*}$ & $9,13 \pm 1,25$ & $8,63 \pm 0,99 *$ \\
\hline Corrida 20m (segundos) & $4,65 \pm 0,51$ & $5,10 \pm 0,55^{*}$ & $4,89 \pm 0,52$ & $4,63 \pm 0,55^{*}$ \\
\hline $\begin{array}{l}\text { Corrida/ Caminhada de } 6 \\
\text { minutos (metros) }\end{array}$ & $1244,86 \pm 216,16$ & $1239,17 \pm 185,21$ & $1197,66 \pm 169,60$ & $1307,13 \pm 154,01^{*}$ \\
\hline
\end{tabular}

\section{Discussão}

O presente estudo conseguiu desenvolver o programa com os jogos pré-desportivos com duração de 52 semanas ressaltando que os voluntários apresentavam sobrepeso e obesidade. A frequência de participação foi acima de $75 \%$ de todos os 38 voluntários do GI, o que é muito importante e, nesse sentido, Guedes (2002) aponta que exercícios de longo prazo podem ser mais efetivos que a curto prazo além de contribuírem para bons resultados tanto na infância e adolescência como na fase adulta.

Crianças e adolescentes precisam receber estímulos para que as habilidades e capacidades motoras possam desenvolver-se de forma natural e eficiente. Assim, programas de esportes e jogos coletivos na infância podem trazer benefícios nas dimensões biopsicossociais desde que sejam trabalhados adequadamente além de ser uma estratégia efetiva na prevenção e tratamento da obesidade (GALATTI; PAES; SEOANE, 2012; GALLAHUE; OZMUN; GOODWAY, 2013; HAYWOOD; GETCHELL, 2016).

A cada modalidade pré-desportiva, desenvolveram-se por 45 sessões, sendo esse número muito superior ao mínimo do recomendado para aprendizagem de uma modalidade esportiva, o que é importante para crianças com sobrepeso e obesidade. Ressalta-se que crianças obesas, muitas vezes, têm pouca habilidade no esporte, ou seja, o excesso de peso corporal 
leva à dificuldade na prática esportiva podendo afastá-las do esporte. O programa realizado pelo GI proporcionou, além da melhora da aptidão física, a aprendizagem de três modalidades esportivas, o que pode contribuir para maior envolvimento com esportes, socialização e uma vida mais ativa (MELLO; LUFT; MEYER, 2004; GONZÁLES; DARIDO; OLIVEIRA, 2014).

Neste estudo, não houve diferenças significativas na antropometria e nas capacidades físicas entre os GC e GI no momento pré, indicando que os grupos eram homogêneos. Os dois grupos participavam das duas aulas semanais de educação física nas mesmas escolas, e não houve orientação nutricional de modo que a participação no programa de exercícios físicos com os jogos pré-desportivos - atletismo, voleibol e basquetebol - foi o único fator de intervenção entre os grupos. O período de férias também não interferiu nesse processo visto que ele deve ocorrer de acordo com o calendário escolar. Ou seja, os voluntários não apresentaram diferenças nas condições antropométricas e capacidades físicas avaliadas após o período de pausa.

Nos voluntários do GC não foram observadas diferenças significativas na massa corporal, IMC, circunferência da cintura e dobra cutânea tricipital pré e pós 52 semanas, indicando que a participação nas duas aulas de educação física escolar não influenciou nas variáveis antropométricas indicadoras da adiposidade corporal. Em relação às capacidades físicas, o presente estudo apontou que as aulas de educação física escolar não foram suficientes para melhorar os níveis de aptidão física dos escolares visto que os resultados indicaram que o GC não teve melhoras significativas nas capacidades físicas analisadas ocorrendo piora na agilidade e na velocidade.

Esses dados estão de acordo com Coledam, Arruda e Oliveira (2012) que estudaram 61 crianças com idade média de 9 anos. Após 12 semanas de intervenção com um programa de exercícios que ocorria duas vezes por semana com 50 minutos de duração, os pesquisadores não observaram diferenças significativas na composição corporal dos participantes. Os autores sugeriram que somente a Educação Física semanal pode não ser suficiente para promover melhoras significativas na composição corporal de crianças e adolescentes.

No GI, ocorreu diminuição no peso corporal (IMC), circunferência da cintura e da dobra cutânea tricipital pré e pós a intervenção indicando que a participação no programa de jogos pré-desportivos de forma complementar às duas aulas de educação física escolar proporcionou benefícios em indicadores da obesidade das crianças com sobrepeso e obesidade. Deve ser destacada a importância da redução da circunferência da cintura, cujo aumento está associado a síndrome metabólica e doenças cardiovasculares.

Espíndola et al. (2014) avaliaram os níveis de aptidão física de seis voluntários com excesso de peso de 7 a 10 de idade durante 8 meses, com um programa ocorria três vezes por semana, totalizando 96 sessões ao todo com exercícios que utilizavam jogos pré-desportivos (futsal, basquete, atletismo) e circuitos. Com isso, eles verificaram redução significativa do IMC dos voluntários. Os dados do presente estudo estão de acordo com os obtidos por Espíndola et al. (2014) mas, esta pesquisa, realizou um programa com duração ainda maior e mais sessões.

Nas capacidades físicas, o GI apresentou melhora nas capacidades relacionadas à saúde (flexibilidade) e ao desempenho esportivo (capacidade cardiorrespiratória), quanto nas relacionadas à força de membros inferiores e superiores, agilidade e velocidade. Não houve diferença significativa apenas na capacidade relacionada à resistência abdominal. Esses resultados apontam que a participação no programa de jogos pré-desportivos complementar as duas aulas de educação física escolar proporcionaram benefícios nas capacidades físicas relacionadas à saúde e ao desempenho esportivo das crianças com excesso de peso corporal.

Generosi (2011) avaliou os efeitos de um programa de exercício físico na aptidão física relacionada à saúde de 17 alunos de 8 a 11 anos de idade em uma escola pública utilizando 
a bateria de avaliações do PROESP-BR (2007). O programa de exercícios foi baseado em atividades esportivas, de corrida e caminhada. Após seis semanas, totalizando 12 sessões, com frequência de 2 vezes por semana, com 40 minutos de duração cada sessão, o autor observou melhora significativa na aptidão cardiorrespiratória, força abdominal e flexibilidade. $\mathrm{O}$ autor ainda sugeriu que programas de exercícios com essa característica devem ser incentivados por melhorarem significativamente a aptidão física dos voluntários estudados e por apresentar baixo custo em sua execução. Esses dados estão de acordo com os achados desta pesquisa ressaltando que o presente projeto teve um tempo muito superior e mais sessões de intervenção.

Espíndola et al. (2014) avaliaram, por meio dos testes do PROESP-BR, seis crianças de 7 a 10 de idade submetidas a intervenção com jogos pré-desportivos durante oito meses e, após análises dos resultados, encontraram melhora na resistência abdominal e agilidade, mas não observaram diferenças significativas nas capacidades flexibilidade, força explosiva de membros superiores, força explosiva de membros superiores e velocidade. Os resultados da presente pesquisa indicam que as 52 semanas de intervenção proporcionaram benefícios em mais capacidades físicas nas crianças com sobrepeso e obesidade.

Após utilizar a bateria de testes preconizada pelo PROESP-BR, Marques et al (2014) avaliaram 39 voluntários com sobrepeso e obesidade, de 7 a 16 anos de idade, sendo 20 participantes do Grupo Intervenção. O programa de exercícios consistia em exercícios com características aeróbias e anaeróbias. Após 16 semanas, com frequência de três vezes por semana e duração de 60 minutos, os autores apontaram que a força de membro superior, agilidade e flexibilidade tiveram melhoras significativas. Segundo os autores, a implantação de programas de exercícios na infância e adolescência deve ser incentivada. Os dados desta pesquisa estão de acordo com os obtidos por Marques et al. (2014), porém o tempo de intervenção nesta foi maior do que naquela.

Deve ser ressaltado que a participação somente nas aulas de educação física escolar não promoveu melhora da aptidão física das crianças do GC de modo que os benefícios observados nas crianças do GI devem ser atribuídos à participação no programa de exercícios físicos com jogos pré-desportivos que complementou as atividades desenvolvidas na educação física escolar. Quando observado os períodos de férias que ocorreram durante o projeto, tanto o GC quanto o GI tiveram o mesmo período, ou seja, o programa de exercícios estava adequado com o calendário escolar e não afetou os resultados apresentados.

Programas de exercícios físicos complementares às aulas de educação física escolar são necessários e este estudo apontou benefícios significativos nas crianças com excesso de peso corporal do GI após 52 semanas de intervenção. A atuação do professor de educação física na área da saúde é fundamental para que, juntamente com equipes interdisciplinares, possam promover melhores condições de saúde e qualidade de vida.

Os resultados deste estudo indicam que, para prevenção da obesidade infantil, as aulas de Educação Física escolar sejam superiores a duas aulas por semana e/ou que sejam complementadas por programas de exercícios físicos. A estratégia de utilização dos jogos prédesportivos com as modalidades atletismo, voleibol e basquetebol mostrou-se adequada para programas de exercícios físicos para crianças do sexo masculino e feminino com excesso de peso corporal.

\section{Conclusão}

As duas aulas de Educação Física por semana realizadas pelas crianças do grupo controle não foram suficientes para promover benefícios em indicadores da obesidade e nas capacidades físicas avaliadas. 
O programa de exercícios físicos com jogos pré-desportivos complementar às duas aulas de Educação Física escolar proporcionaram às crianças do grupo intervenção melhora em indicadores da adiposidade, das capacidades relacionadas à saúde e ao desempenho esportivo.

A proposta de intervenção do presente estudo utilizando os jogos pré-desportivos (atletismo, voleibol e basquetebol) complementares às duas aulas de educação física escolar foi eficaz para melhora da aptidão física das crianças com sobrepeso e obesidade indicando ser uma estratégia interessante na prevenção da obesidade infantil.

\section{Agradecimentos}

À Coordenação de Aperfeiçoamento de Pessoal de Nível Superior (CAPES) pela taxa de doutorado.

\section{Colaboração dos autores}

Todos os autores participaram do planejamento e desenvolvimento do estudo, o que envolve coleta, tratamento e análise de dados, elaboração, redação e revisão.

\section{Conflito de interesse}

Não há conflitos de interesse.

\section{Referências}

ALVES, J.G.B et al. Efeito do exercício físico sobre peso corporal em crianças com excesso de peso: Ensaio clínico comunitário randomizado em uma favela no Brasil. Cadernos de saúde pública, v.34, n.38, p. 353-359, 2008.

AMARAL, A.P.A; PALMA, A.P. Perfil epidemiológico da obesidade em crianças: Relação entre televisão, atividade física e obesidade. Revista Brasileira de Ciências do Movimento. v.4, n.9, p. 19-24, 2001.

AMERICAN COLLEGE OF SPORTS MEDICINE. Diretrizes do ASCM para os testes de esforço e sua prescrição. Rio de Janeiro: Guanabara Koogan, 2014.

AMERICAN COLLEGE OF SPORTS MEDICINE. Aptidão física na criança e no adolescente. Revista Brasileira de Medicina do Esporte. v.3, n. 2, p. 39-40, 1997.

ASSOCIAÇÃO BRASILEIRA DE ESTUDOS DA OBESIDADE E SÍNDROME METABÓLICA. Diretrizes Brasileiras de Obesidade 2009/2010. 3. ed. Itapevi, 2009.

BRASIL. Ministério da Saúde. Gabinete Ministerial. Portaria n. 154 de 24 de janeiro de 2008: Cria os núcleos de apoio à saúde da família (NASF). Diário Oficial da União, Brasília, p. 38-42, 2008.

BRASIL. Ministério da Saúde. Gabinete Ministerial. Portaria n. 3.124 de 28 de dezembro de 2012. Redefine os parâmetros de vinculação dos Núcleos de Apoio à Saúde da Família (NASF) modalidades 1 e 2 às Equipes Saúde da Família e/ou Equipes de Atenção Básica para populações específicas, cria a Modalidade NASF 3, e dá outras providencias. Diário Oficial da União, Brasília, 2012. 
BRASIL. Ministério da Saúde. Diretrizes do NASF: Núcleo de Apoio a Saúde da Família. Cadernos de Atenção Básica, 2010.

BRASIL. Ministério da Saúde. Vigitel Brasil 2016. Vigilância de fatores de risco e proteção para doenças crônicas por inquérito telefônico. 2016.

COLEDAM, D.H.C; ARRUDA, G.A; OLIVEIRA, A.R. Efeitos de um programa de exercícios no desempenho de crianças nos testes de flexibilidade e impulsão vertical. Revista Motriz. v.18, n.3, p. 515-525, 2012.

COSTA, R.F. Composição corporal: teoria e prática da avaliação. Barueri: Manole, 2001.

DARIDO, S.C. Educação Física na escola: questões e reflexões. Rio de Janeiro: Guanabara Koogan, 2003.

ESPÍNDOLA, J. A et al. Efeitos do exercício físico na aptidão física de crianças com sobrepeso. Revista Conscientiae Saúde, v.13, n. 2, p. 281-288, 2014.

FRISANCHO, A.R. New norms of upper limb fat and muscle áreas for assessment of nutritional status. The American Journal of Clinical Nutrition. Estados Unidades, v.34, n.1, p. 2540-2545, 1981.

GALATTI, L.R; PAES, R; SEOANE, A.M. Pedagogia do esporte e obesidade: Perspectiva para um estilo de vida saudável com base na adequada iniciação esportiva na infância. Pensar a Prática. Goiás, v.15, n.2, p. 484-498, 2012.

GALlAHUE, D.L; OZMUN, J.C; GOODWAY, D. J. Compreendendo o Desenvolvimento Motor: Bebês, Crianças, Adolescentes e Adultos. 7. ed. Porto alegre: Artmed; 2013.

GENEROSI, R. A. Efeitos de um programa de exercício físico em componentes da aptidão física relacionada à saúde de escolares. 2011. Dissertação (Mestrado) - Programa de Pós Graduação em Ciências do Movimento Humano, Universidade Federal do Rio Grande do Sul, Porto Alegre, 2011.

GONZÁLES, J.F; DARIDO, S.C; OLIVEIRA, A.A.B. (Org.). Esporte de marca e com rede divisória ou muro/ parede de rebote: Badminton, peteca, tênis de campo, tênis de mesa, voleibol e atletismo. Maringá: Eduem, 2014.

GONZÁLES, J.F; DARIDO, S.C; OLIVEIRA, A.A.B. (Org). Esportes de Invasão: Basquetebol, futebol, futsal, handebol e ultimate frisbee. Maringá: Eduem, 2014.

GUEDES, D.P. Educação para saúde mediante programas de Educação Física Escolar. Revista Motriz. v.5, n.1, p. 1-6, 1999.

GUEDES, D. P. Programas de controle do peso corporal: Atividade Física e Nutrição. Revista Mineira de Educação Física. Minas Gerais, v.101, n.1, p. 64-90, 2002.

GUEDES, D.P. Atividade física, aptidão física e saúde. Orientações básicas sobre atividade e saúde para profissionais das áreas da Educação e Saúde. Brasília: Ministério da Saúde e Ministério da Educação e do Desporto, 1996. 
HAYWWOOD, K.M; GETCHELL, N. Desenvolvimento motor ao longo da vida. 6. ed. Porto Alegre: Artmed, 2016.

INSTITUTO BRASILEIRO DE GEOGRAFIA E ESTATÍSTICA- IBGE. Pesquisa de orçamentos familiares 2008-2009: Antropometria e análise do estado nutricional de crianças e adolescentes no Brasil. Disponível em: www.ibge.gov.br. Acesso em: 10 de jan. de 2016.

LIMA, S.C.V.C et al. Perfil lipídico e peroxidação de lipídeos no plasma de crianças e adolescentes com sobrepeso e obesidade. Jornal de Pediatria, v.80, n.1, p. 23-28, 2004.

MARQUES, K.C et al. Programa de Intervenção com exercícios físicos melhora indicadores de aptidão física relacionada à saúde e ao desempenho motor em escolares com sobrepeso e obesidade. Revista Cinergis, Santa Cruz do Sul, v.15, n.3, p. 119-122, 2014.

MELLO, E.D; LUFT, V.C; MEYER, F. Obesidade Infantil: como podemos ser eficazes? Jornal de Pediatria. Brasil, v.80, n3, p. 174-182, 2004.

MOTA, J.L.P et al. Análise do perfil antropométrico e nível de atividade física de alunos de $6^{\mathrm{a}}$ e $7^{\text {a }}$ séries do ensino fundamental de Nova Campina/SP. Revista Brasileira de Obesidade, Nutrição e Emagrecimento, v.9, n.50, p. 51-58, 2015.

OLIVEIRA, A.M.A et al. Sobrepeso e obesidade infantil: Influência dos fatores biológicos e ambientais em Feira de Santana, BA. Arquivos Brasileiros de Endocrinologia e Metabologia, v.42, n.2, p. 144-150, 2003.

OLIVEIRA, C. L et al. Obesidade e síndrome metabólica na infância e adolescência. Revista de Nutrição. São Paulo, v.17, n.2, p. 237-245, 2004.

OLIVEIRA, C.L; FISBERG, M. Obesidade na infância e adolescência uma verdadeira epidemia. Arquivos Brasileiros de Endocrinologia e Metabologia, v.42, n. 2, p. 107-108,2003.

PROJETO ESPORTE BRASIL (PROESP-BR). Avaliação da aptidão física relacionada à saúde (APFS). Disponível em: www.proesp.ufrgs.br. Acesso em: 25 ago. 2014.

PROPOSTA CURRICULAR DO ESTADO DE SÃO PAULO. Educação Física. São Paulo: Secretaria Estadual de Educação, 2008.

SILVA, V.S et al. A importância da educação física escolar no desenvolvimento motor de crianças nos anos iniciais do ensino fundamental. Visão dos responsáveis. Revista EF Desportes, v.16, n.156, 2011.

SOCIEDADE BRASILEIRA DE MEDICINA DO ESPORTE. Atividade física e saúde na infância e adolescência. Revista Brasileira de Medicina do Esporte, v.4, n. 4, p. 107-109, 1998.

SOCIEDADE BRASILEIRA DE PEDIATRIA. Departamento Científico de Nutrologia. Obesidade na infância e adolescência- Manual de Orientação. Disponível em: www.sbp.com.br. Acesso em: 20 mar. 2016. 
Recebido em: 05/06/2018

Revisado em: 31/07/2018

Aprovado em: 10/08/2018

Endereço para correspondência:

brunovespasiano@msn.com

Bruno de Souza Vespasiano

Universidade Metodista de Piracicaba,

Faculdade de Ciências da Saúde, Educação Física.

Rodovia do Açúcar, $\mathrm{n}^{\circ} 7000$

Taquaral

13400911 - Piracicaba, SP - Brasil 